\title{
The role of the third component in ternary organic solar cells
}

Nicola Gasparini ${ }^{1}$, Alberto Salleo ${ }^{2}$, Iain McCulloch ${ }^{1,3}$ and Derya Baran ${ }^{1 *}$

\footnotetext{
${ }^{1}$ King Abdullah University of Science and Technology (KAUST), Division of Physical Sciences and Engineering (PSE), KAUST Solar Center (KSC), Thuwal, Saudi Arabia.

${ }^{2}$ Department of Materials Science and Engineering, Stanford University, Stanford, CA, USA.

${ }^{3}$ Department of Chemistry and Centre for Plastic Electronics, Imperial College London, London, UK.

*e-mail: derya.baran@kaust.edu.sa
}

\section{ToC blurb}

Adding a third component into a binary blend is a promising strategy to simultaneously improve all photovoltaic parameters in organic solar cells. In this Review, we discuss the role of the third component in influencing the energetics, charge-carrier recombination and stability in ternary solar cells.

\begin{abstract}
Ternary organic solar cells (TSCs) contain a single three-component photoactive layer with a wide absorption window, which is obtained without the need for multiple-stacks. Subsequently, TSCs have attracted great interest in the photovoltaics field. Through careful selection of the three (or more) active components that form the photoactive layer, all photovoltaic parameters can be simultaneously enhanced within a TSC - a strategy that has resulted in record efficiencies for single-junction solar cells. In this Review, we outline key developments in TSCs, with a focus on the central role of the third component in achieving record efficiencies. We analyse the effects of the third component on the nanomorphology of the bulk heterojunction and the photovoltaic parameters of TSCs. Moreover, we consider the charge-transfer and/or energy-transfer mechanisms and nanomorphology models that govern the operation of TSCs. We consider both polymer and small-molecule donors as well as
\end{abstract}


fullerenes and recently developed non-fullerene acceptors. In addition, we summarize the recent success of TSCs in mitigating the stability issues of binary solar cells. Finally, we provide a perspective on the advantages of ternary blends and suggest design strategies for highly efficient and stable devices for commercial photovoltaics.

\section{[H1] Introduction}

The advent of solar cells is commonly associated with the discovery of the photoelectric effect in 1839 , when Becquerel observed a photocurrent upon the irradiation of platinum electrodes with light ${ }^{1}$. Almost a century later, photoconductivity was first observed from an organic compound, anthracene ${ }^{2}$. Since the initial observation of photoconductivity, interest in organic semiconductors has increased steadily, and today, considerable research effort is focused on the development of organic lightemitting diodes, transistors, photodetectors and solar cells as future optoelectronic devices ${ }^{3-9}$. Among these optoelectronic devices, organic solar cells (OSCs) are strong candidates for energy-harvesting applications, in which light is converted into electricity with no carbon footprint ${ }^{10-17}$. The semitransparency, solution processability, freedom of form and low weight make OSCs viable contenders for low-cost, high power-to-weight ratio technologies for future electricity generation ${ }^{18-20}$.

OSCs were first realized in 1986, when Tang demonstrated that a bilayer comprising an electron donor and electron acceptor could be used as a photoactive layer to produce photocurrent ${ }^{21}$. The bilayer architecture, however, has a limited interfacial area, which results in a low photocurrent owing to the well-known exciton bottleneck. The problem of limited interfacial area has been mitigated by the introduction of the bulk-heterojunction (BHJ) structure, which was obtained independently by the Heeger and Friend groups in 1995 by blending polymer/fullerene and polymer/polymer mixtures, respectively ${ }^{22,23}$. The invention of the $\mathrm{BHJ}$ concept was a milestone in the organic photovoltaics (OPVs) field, and consequently, power conversion efficiencies (PCEs) of OSCs have increased to $>14 \%$ in the past three decades in single-layer BHJ devices. By increasing the interfacial area between the electron donor and electron acceptor, BHJ solar cells overcome the issues of short exciton diffusion length, limited exciton lifetime and charge separation that limit bilayer junctions, enabling efficient, thick and large-area OSCs that are one step closer to large-scale production and commercialization.

Although the introduction of the BHJ structure has enabled great improvements in efficiency, it has also focused research efforts towards the development of increasingly complex and sophisticated 
morphologies, as the intricate BHJ morphology has a key role in governing the PCE in OSCs. There are various types of donor materials, from the work-horse homo-polymer poly(3-hexylthiophene) (P3HT) to more recent push-pull type hetero-polymers (which are commonly known by their abbreviations, such as, PTB7 (ref. ${ }^{24}$ ), PTB7-Th ${ }^{25}$, PffBT4T-2OD ${ }^{26}$, P3TEA $^{27}$ and PBDB-T ${ }^{28}$ ) and small-molecule donors (for example, p-DTS(FBTTh $)_{2}\left(\right.$ ref. $\left.^{29}\right)$ and DR3TSBDT ${ }^{30}$ ) that continue to push record efficiencies (Fig. 1). There have been intensive synthetic efforts to identify the ideal polymer or small-molecule donor, with a focus on precise energy-level matching with the complementary acceptor molecules and optimization of the resultant nanomorphology created through thin-film fabrication processing ${ }^{31}$. One of the main reasons for the initial focus on donors was the ease of tuning their molecular electronic energy levels in comparison to those of soluble fullerene derivatives (such as [6,6]-phenyl- $\mathrm{C}_{61}$ butyric acid methyl ester $\left(\mathrm{PC}_{60} \mathrm{BM}\right)$ or $[6,6]$-phenyl- $\mathrm{C}_{71}$ butyric acid methyl ester $\left.\left(\mathrm{PC}_{70} \mathrm{BM}\right)\right)$, which were initially the sole acceptor candidates. These fullerene-derived acceptors usually absorb only in the high-energy region of the visible spectrum, leaving a large absorption window at lower energies. Furthermore, structural modification of these fullerene derivatives revealed that they have relatively fixed electron affinities (EAs) and ionization potentials (IPs), which hinders access to devices with a high open-circuit voltage $\left(V_{\text {oc }}\right.$; that is, $\left.>1 \mathrm{~V}\right)$ and high photocurrent. Within the past 10 years, non-fullerene acceptor (NFA) materials, including both smallmolecule acceptors and polymer acceptors, have emerged as serious competitors to fullerenes as electron-acceptor materials for OPV devices ${ }^{32-40}$. Unlike fullerene derivatives, NFAs consist of either push-pull calamitic-type moieties (for example, IDTBR, ITIC and IEICO) ${ }^{28,36,41,42}$ or naphthalenediimide (NDI) ${ }^{43,44}$ or perylenediimide (PDI) ${ }^{27,45}$ cores (Fig. 2). The energy levels of NFAs can be tuned through modifications to the conjugated skeletons. For example, decreasing the electronwithdrawing strength of the peripheral units decreases the EA (versus vacuum), resulting in high $V_{\mathrm{oc}}$ values. Moreover, the solubility and blend morphology of NFAs can be controlled through side-chain engineering and their bandgap $\left(E_{\mathrm{g}}\right)$ can be adjusted to harvest photons in a region of the solar spectrum that is complementary to the absorption region of the donor. These advantages of NFAs have contributed to champion OSCs with PCEs of up to $10.1 \%{ }^{46}$ and $14.6 \%{ }^{47}$ for polymer acceptors and small-molecule acceptors, respectively. However, although these efficiencies have surpassed those obtained in devices made with fullerene acceptors, challenges in single-junction binary BHJ OSCs still remain in terms of controlling the nanomorphology, minimizing recombination and increasing the long-term stability of devices ${ }^{48-56}$. 
Over the past decade, numerous strategies have been proposed to improve upon the PCE of single-junction binary devices. Among the strategies, multi-junction (such as tandem) solar cells, in which two or more sub-active layers are monolithically or vertically connected with interfacial recombination layers, have been extensively explored ${ }^{57-64}$. Tandem devices contain two sub-active layers with different absorption windows to increase either the voltage or current, depending on how the sub-active layers are electrically connected. Although record efficiencies have been reported for tandem solar cells, the fabrication of these devices with optimal recombination layers between the subactive layers is challenging. Ternary organic solar cells (TSCs), in which a single active layer with a wide absorption window is created without the need for a multiple-stacked interfacial layer, have attracted great interest in the area of photovoltaics owing to the simple device design ${ }^{35,65-69}$. Blending three light-absorbing semiconductor materials (where the third component has the minority ratio in the blend and is defined as the guest) or more components (with at least one donor and one acceptor as the host system) can lead to more efficient sunlight harvesting ${ }^{70-72}$. An increase in the efficiency of sunlight harvesting translates to a higher short-circuit current density $\left(J_{\mathrm{sc}}\right)$ in the solar cell devices while maintaining the simplicity of the single-junction architecture. Moreover, by carefully selecting the components on the basis of the relative energies of their frontier orbitals, it is possible to tune the $V_{\text {oc }}$ through the formation of an energy cascade alignment ${ }^{35,73-75}$. Furthermore, introducing an extra component can help to overcome transport and/or charge-recombination issues associated with binary devices. For example, the addition of a third component can decrease the density of trap states and increase charge mobility, which results in ternary blends with enhanced fill factors (FFs) and enables thick active layers $(>200 \mathrm{~nm})^{65,76,77}$. Thorough understanding of the fundamental mechanisms (such as charge and energy transfer) that govern the charge dynamics and $V_{\mathrm{oc}}$ in ternary blends has enabled the fabrication of TSCs that demonstrate simultaneous enhancement of all photovoltaic parameters $\left(J_{\mathrm{sc}}, V_{\mathrm{oc}}\right.$ and FF), resulting in a record single-junction PCE of $14 \%$.

To date, reviews on ternary blends have largely served to describe the chemical structures of donors and/or acceptors and the energetic processes ${ }^{67-69,78-80}$. In this Review, we outline the most significant recent developments in ternary BHJ OSCs with a focus on the key role of the third component. First, we provide a brief history of OSCs and an overview of the pioneering reports on single-junction TSCs. Second, we discuss the existing operating modes of TSCs (which are governed by the nanomorphology and energetics of the ternary blend) that account for the role of the third component in TSCs. The models used to explain the operation of TSCs typically describe the location 
of the third component in the blend and the effect of mixing the third material with one of the other components (donor or acceptor). Third, we assess the role of the third component in influencing the electronic energy level alignment, spectral coverage, charge recombination and nanomorphology of ternary blends and devices ${ }^{35,70-75,81-89}$. We also consider the stability of the active-layer materials and devices as well as the recent progress in the development of ternary blends to help overcome the degradation and/or reliability issues of binary solar cells ${ }^{35,90}$. The scalability of ternary devices that have yielded record device PCEs $(>14 \%)$ and environmentally friendly processing opportunities are also briefly discussed ${ }^{65,76,77,91-94}$. Finally, we provide a critical perspective on the advantages of ternary blends and devices along with opportunities for improvements and suggest design strategies for highly efficient and stable OSCs for commercially viable photovoltaics.

\section{[H1] Operating modes of TSCs}

The BHJ is the key component of an OSC device stack (Fig. 3a). Binary BHJ solar cells consist of two components: a primary electron donor $\left(\mathrm{D}\right.$ or $\left.\mathrm{D}_{1}\right)$ and a primary electron acceptor $\left(\mathrm{A}\right.$ or $\left.\mathrm{A}_{1}\right)$ that are blended in a common solvent at various D:A ratios. The addition of a third component (a secondary donor, $\mathrm{D}_{2}$, or secondary acceptor, $\mathrm{A}_{2}$ ) into the binary blend, which serves as the host system, results in a ternary blend. The TSCs resulting from such combinations display properties that are more than a simple superposition of those of the individual components. Depending on the energetics and phase structure of the third component, the location of the third component in a BHJ determines the primary operating mode of a 'TSC'. For example, the energy cascade alignment ${ }^{84,95}$ and energy-transfer and/or charge-transfer mechanisms ${ }^{85,96}$ are operating modes governed by the energetics whereas the operating modes in the 'alloy' and 'parallel-like ${ }^{97}$ models is primarily determined by the nanomorphology. The BHJ of the ternary blends can be classified into five main categories according to the location of the third component: embedded (or dissolved) in the donor phase (Fig. 3b), embedded (or dissolved) in the acceptor phase (Fig. 3c), residing between the donor and acceptor phases (Fig. 3d), co-crystallized with the donor moiety (Fig. 3e) or co-crystallized with the acceptor moiety (Fig. 3f). However, in practice, the BHJ of ternary blends can comprise one or more of these BHJs depending on the ratio of $\mathrm{D}_{2}$ or $\mathrm{A}_{2}$ to the binary host system, and therefore more than one operating mode can be adopted.

In a typical TSC, a small amount of third component $\left(\mathrm{D}_{2}\right.$ or $\left.\mathrm{A}_{2}\right)$ is added into a binary blend. Depending on the position of the third component in the bulk, the nanomorphology of $\mathrm{D}_{1}$ or $\mathrm{A}_{1}$ usually improves the ternary BHJ through enhanced crystallization of one or both of the binary components, 
increased pure domain sizes of the binary blends and/or extended coherence length of these domains. This improved microstructure often has a positive effect on charge transport and the recombination behaviours in ternary OSCs compared to binary devices, resulting in an increase in the FF and $J_{\text {sc. }}$ Depending on the energetics of $\mathrm{D}_{2}$ and $\mathrm{A}_{2}$, different operating modes are proposed. The energy cascade model (Fig. 4a) is one of the operating modes of TSCs. Within the energy cascade model, the IP and EA levels of the third component are in between the IP and EA levels of $\mathrm{D}_{1}$ and $\mathrm{A}_{1}$, respectively, such that the $\mathrm{D}_{2}$ or $\mathrm{A}_{2}$ material facilitates the transport of holes to the anode and electrons to the cathode ${ }^{96}$. The efficiency of the charge-transfer process increases when the third component is located at the donor/acceptor interface ${ }^{99}$. Energy transfer, also known as Förster resonance energy transfer (FRET), can compete with charge transfer. Efficient FRET requires overlap of the emission spectrum of $\mathrm{D}_{1}$ (or $\left.\mathrm{A}_{1}\right)$ and the absorption spectrum of the third component, as well as a short distance $(<10 \mathrm{~nm})$ between the two components. Both charge and energy transfer can simultaneously occur in ternary systems ${ }^{83,70}$. For example, energy transfer from $\mathrm{D}_{1}$ and $\mathrm{D}_{2}$ may favour the splitting of excitons located further away from the $\mathrm{D}_{1} / \mathrm{A}$ interface, with subsequent charge transfer occurring between the two donor materials following exciton splitting at the $\mathrm{D}_{2} / \mathrm{A}$ interface.

The energy cascade model, however, cannot predict the sole evolution of $V_{\mathrm{oc}}$ and $J_{\mathrm{sc}}$ in $\mathrm{D}_{1}: \mathrm{D}_{2}: \mathrm{A}$ and $\mathrm{D}: \mathrm{A}_{1}: \mathrm{A}_{2}$ ternary devices. There are therefore several questions regarding the operating modes of TSCs and, in particular, the spatial organization of the donor/acceptor interfaces ${ }^{74,84,95,97,98}$. In this regard, two dominant nanomorphology models have been proposed to explain the evolution of the $V_{\text {oc }}$ and $J_{\text {sc }}$ in TSCs: the parallel-like model and the alloy model. A parallel-like BHJ is different from that in typical TSCs, in which small amounts of the third component are used, and uses $\mathrm{D}_{1}: \mathrm{D}_{2}\left(\right.$ or $\left.\mathrm{A}_{1}: \mathrm{A}_{2}\right)$ mixtures with different bandgaps at any composition regardless of their IP or EA levels. The parallel-like BHJ is therefore analogous to the parallel connections between sub-cells in a tandem architecture, in which the complimentary sub-cells absorb light, split excitons and transport holes. In the parallel-like model, the excitons generated in each individual donor material migrate to the respective donor/acceptor interface and dissociate into free charge carriers. Subsequently, the holes are transported in the donor matrix to the anode and the electrons to the cathode through the acceptor matrix. With a parallel-like BHJ, the $V_{\text {oc }}$ of the ternary blend lies between the values of the binary blends; this is in contrast to the energy cascade model, in which the $V_{\mathrm{oc}}$ values of ternary devices are often close to the lowest $V_{\text {oc }}$ value of the binary devices. The $J_{\mathrm{sc}}$ values of parallel-like TSCs are nearly the sum of the two binary cells, proving that the sub-cells work independently in the ternary composite, analogous to tandem architectures. By 
contrast, in the alternative alloy model, it is proposed that the energy levels of $D_{1}: D_{2}$ or $A_{1}: A_{2}$ materials in the ternary blends form electronic alloys that have a single IP or EA, respectively ${ }^{74,98}$. The formation of such alloys requires good miscibility of the materials used in the ternary blends. It is important to note that all the aforementioned models can coexist, which complicates the prediction of the mechanisms that govern the photovoltaic processes. Owing to the limited examples of parallel-like TSCs, there has not yet been a thorough study of this model. Moreover, the alloy model has failed to explain the $V_{\text {oc }}$ evolution in several TSCs, especially for fullerene-based TSCs with high ratios of a third component ${ }^{89,99}$.

\section{[H1] The role of the third component}

The discovery of novel organic materials together with tailored morphologies and device engineering has led to OSCs with PCEs $>14 \%^{101,102}$. Moreover, understanding of the microstructure and charge recombination dynamics has enabled the optimization of $\mathrm{FF}$ values to $>80 \% 47$. However, despite these significant improvements, the PCE of OPV devices is still lower than that of commercial inorganic solar cells. The main limitation on the PCE of OPV devices is due to the intrinsically narrow spectral absorption range of organic materials compared with the absorption range of inorganic semiconductors, such as silicon or hybrid perovskites. The limited absorption range makes it challenging to fully cover the solar spectrum with a single-junction device without sacrificing $V_{\text {oc. }}$ Furthermore, owing to the low carrier mobility and short lifetime of the photoactive materials, OSCs have a limited active-layer thickness, typically $\sim 100 \mathrm{~nm}$. The limited thickness of the active layer impedes the 'lab-to-fab' transition for reliably and inexpensively scaling up production, which requires active-layer thicknesses of $\sim 200-300 \mathrm{~nm}$. In the following, we focus on strategies for improving one or more of the

photovoltaic parameters of ternary devices compared with those of the binary cells (Fig. 4, 5). The strategies for increasing $J_{\mathrm{sc}}$ and $V_{\mathrm{oc}}$ as well as controlling charge recombination are discussed together with consideration of the change in nanomorphology upon addition of the third component. The representative donor and acceptor materials are shown in Fig. 1 and Fig. 2, respectively.

\section{[H2] Energetics and spectral coverage.}

In the first report on using a ternary blend to increase the $J_{\mathrm{sc}}$ of a BHJ OSC, a near-infrared (IR) lowbandgap polymer, PCPDTBT, was added as the third component to the well-known binary blend $\mathrm{P} 3 \mathrm{HT}: \mathrm{PC}_{60} \mathrm{BM}^{103}$. The resulting ternary blend features an additional long-wavelength absorption (up to $800 \mathrm{~nm}$ ) compared with the absorption of the binary blend (which extends up to $650 \mathrm{~nm}$ only). As a 
consequence, devices based on P3HT:PCPDTBT:PC ${ }_{60} \mathrm{BM}$ outperformed those based on the binary P3HT:PC ${ }_{60} \mathrm{BM}$ blend with a $12 \%$ relative increase in the PCE (Table 1). An even better result (a 29\% relative increase in PCE) was obtained when a modified version of PCPDTBT, Si-PCPDTBT, was introduced as the third component into $\mathrm{P} 3 \mathrm{HT}: \mathrm{PC}_{60} \mathrm{BM}^{71}$. More recently, the development of novel midbandgap and low-bandgap polymers, such as PTB7, PTB7-Th, PBDB-T and PffBT4T-2OD (Fig. 1), has yielded record PCEs of $>10 \%$ for binary devices. For many years, blends based on PTB7 (or PTB7$\mathrm{Th}): \mathrm{PC}_{70} \mathrm{BM}$ were used as reference cells for TSCs owing to their low charge-carrier mobility and trapassisted recombination $65,76,78,104-107$. For example, the polymer $\mathrm{PID}_{2}$ can be introduced as a $\mathrm{D}_{2}$ component that forms an energy cascade alignment between the IP and EA levels of PTB7 and $\mathrm{PC}_{70} \mathrm{BM}$, respectively ${ }^{85}$. A small-molecule donor, p-DTS $\left(\mathrm{FBTTH}_{2}\right)_{2}$, has also been used as a $\mathrm{D}_{2}$ component $^{89}$. The $\mathrm{D}_{1}: \mathrm{D}_{2}$ :A blends with p-DTS $\left(\mathrm{FBTTH}_{2}\right)_{2}$ demonstrated a concomitant increase in the $J_{\mathrm{sc}}$ and FF. This increase is attributed to the complementary absorption of the donor molecules and, benefiting from the energy cascade alignment, improved charge separation and transport, which leads to decreased charge recombination. Importantly, it was postulated that an alloy forms between the $\mathrm{D}_{1}$ and $\mathrm{D}_{2}$ materials, which results not only in a decrease in charge recombination but also in a highly ordered face-on orientation of PTB7 with respect to the substrate. The face-on orientation enables favourable interactions for efficient charge transfer and limits charge-carrier recombination, which improves charge transport and subsequently led to the high FF obtained as well as the first reported PCE of $>10 \%$ for a TSC.

State-of-the-art NFAs, such as IDTBR and ITIC derivatives ${ }^{28,36}$, have been designed to complement the absorption of the donor materials and exhibit panchromatic absorption from the visible to the IR region of the solar spectrum. With a state-of-the-art low-bandgap NFA, IEICO-4F (Fig. 2), record $J_{\mathrm{sc}}$ values of $>27 \mathrm{~mA} \mathrm{~cm}^{-2}$ have been reported ${ }^{108}$. However, owing to the low absorption coefficients of the donor materials, the external quantum efficiencies (EQEs) obtained in the 400$600 \mathrm{~nm}$ region are still far below those measured in the longer wavelength regions, in which NFA absorption dominates. For this reason, the ternary strategy has also been used to sensitize the highenergy region through the introduction of $\mathrm{D}_{2}$ or $\mathrm{A}_{2}$ materials (Fig. 4b). For example, PTB7-Th was added as a $\mathrm{D}_{2}$ component into a binary PBDB-T:IEICO-4F blend, resulting in the PCE increasing from $10.2 \%$ to $11.6 \%$, mainly due to the increase in $J_{\mathrm{sc}}$ from $22.8 \mathrm{~mA} \mathrm{~cm}^{-2}$ in the binary blend to $24.1 \mathrm{~mA} \mathrm{~cm}^{-2}$ in the $\mathrm{D}_{1}: \mathrm{D}_{2}: \mathrm{A}$ ternary blend ${ }^{72}$. PTB7-Th not only increased photon harvesting but also increased the efficiency of energy transfer between the two donors. A similar outcome was obtained 
with a $\mathrm{D}: \mathrm{A}_{1}: \mathrm{A}_{2}$ ternary blend, in which the $\mathrm{A}_{2}$ component was a PDI-based NFA that was used in combination with a D:A $($ PDBT-T1:ITIC-Th) binary blend to increase the absorption of the binary blends in the $400-600 \mathrm{~nm}$ region ${ }^{104}$.

Despite the great progress made in the OPV field, in particular to achieve a high $J_{\mathrm{sc}}$, the relatively large voltage loss, defined as $\left(E_{\mathrm{g}} / q\right)-V_{\mathrm{oc}}=0.6 \mathrm{~V}$ (where $q$ is the elementary charge), limits the PCE to $<20 \%$. High $V_{\mathrm{oc}}$ values are associated with relatively low $J_{\mathrm{sc}}$ values and EQE values $<70 \%{ }^{56}$. However, this trade-off can be alleviated by adopting the ternary strategy. The introduction of a third component that has suitable energy levels to form an energy cascade alignment with the binary components, in principle, leads to an increase in $V_{\mathrm{oc}}$ without the compromise of a reduction in $J_{\mathrm{sc}}$ through the creation of efficient charge-extraction channels ${ }^{71,85}$ (Fig. 4a). Fullerene derivatives such as $\mathrm{IC}_{60} \mathrm{BA}^{109}$ that have an EA higher than that of $\mathrm{PC}_{70} \mathrm{BM}$ have been used as an $\mathrm{A}_{2}$ component to increase the $V_{\mathrm{oc}}$ of binary devices. For example, a simultaneous increase in both $J_{\mathrm{sc}}$ and $V_{\mathrm{oc}}$ was observed when $\mathrm{IC}_{60} \mathrm{BA}$ was added into a PTB7:PC $70 \mathrm{BM}$ binary blend ${ }^{98}$. In this case, the formation of an energy cascade alignment between the components increased the $V_{\mathrm{oc}}$ by $\sim 20 \mathrm{mV}$ on going from the binary to ternary blend. The bridging role of $\mathrm{IC}_{60} \mathrm{BA}$ between the $\mathrm{D}$ and $\mathrm{A}_{1}$ components improves charge transfer at the $\mathrm{D} / \mathrm{A}_{1}$ interface by reducing the density of traps without disrupting the nanomorphology. Similar observations have been reported in all-polymer ternary blends. For example, the $V_{\mathrm{oc}}$ of PTB7Th:PND(2OD)2T devices increases upon addition of PBDD-ff4T, which is attributed to the formation of an energy cascade alignment between the components ${ }^{110}$.

Materials and nanostructure engineering in binary cells are often optimized by taking into account the well-known trade-off between $J_{\mathrm{sc}}$ and $V_{\mathrm{oc}}$. For TSCs, the addition of a third component introduces a new trade-off between FF and $V_{\mathrm{oc}}$ that needs to be considered. The $V_{\mathrm{oc}}$ is predominantly determined by the energy of the charge-transfer state at the donor/acceptor interface, corrected by a recombination term ${ }^{111-113}$. A change of one order of magnitude in the recombination rate changes the $V_{\text {oc }}$ by only $\sim 60 \mathrm{meV}$. It has been shown that the photovoltage is governed by the energetics of the interface at which charges are generated and is nearly unaffected by the IP and EA levels of the transport layers ${ }^{111-113}$. Because the energy of the charge-transfer state is heavily influenced by the energetics and local structure at the donor/acceptor interface, the introduction of a third component at that interface can strongly affect the $V_{\mathrm{oc}}$ (ref. ${ }^{75}$ ). Note that the most studied OPV active layer, P3HT:PC ${ }_{60} \mathrm{BM}$ can be regarded as a ternary cell in which P3HT forms crystalline domains and also a disordered mixed phase with $\mathrm{PC}_{60} \mathrm{BM}$, which leads to a minimum of three phases (pure P3HT, pure 
$\mathrm{PC}_{60} \mathrm{BM}$ and the amorphous mixed phase) ${ }^{111}$. Increasing the amorphous nature of the donor/acceptor interface increases the $V_{\text {oc }}$ owing to the disorder-induced increase in the IP of P3HT and decrease in the $\mathrm{EA}$ of $\mathrm{PC}_{60} \mathrm{BM}^{75}$. The possible increase in recombination rate owing to disorder does not typically mitigate the effect of the disorder-induced increase in the energy of the charge-transfer state on $V_{\text {oc. }}$. Hence, increasing disorder at the donor/acceptor interface is beneficial in obtaining a higher $V_{\text {oc }}$.

\section{[H2] Charge recombination and nanomorphology.}

Mixing in new components changes the morphology (both in the bulk and at the nanoscale) of the binary blend, possibly altering charge-transport and recombination paths and thereby affecting the charge-extraction efficiency. Furthermore, key parameters that govern PCE, such as FF and $V_{\mathrm{oc}}$, depend strongly on the nanomorphology of the active layer and will therefore be affected by the introduction of additional components ${ }^{91}$. Indeed, the impact of morphology on the PCE of OPVs is not completely understood in binary cells; in ternaries and cells with even more components, our understanding of the role of morphology is still nascent. At present, it is difficult to predict a priori the photovoltaic parameters of a ternary blend based on the energetics of the components owing to the formation of varied nanostructures. This difficulty has prevented the emergence of generalized optimization guidelines for ternary blends.

When choosing a third component, the term 'compatibility' is often mentioned, albeit somewhat vaguely. Physical compatibility has come to signify the ability of the two materials to mix without substantially altering the crystallinity and texture of the pure phases. For example, $\mathrm{A}_{2}$ is considered to be physically compatible with the $\mathrm{D}: \mathrm{A}_{1}$ blend if the addition of $\mathrm{A}_{2}$ does not alter the nanomorphology of the $\mathrm{D}: \mathrm{A}_{1} \mathrm{BHJ}$. Chemical compatibility, however, has come to signify something close to miscibility. As a result, compatibility of the third component with the binary components is an important factor in determining the final morphology of the active layer. From the standpoint of measureable properties, compatibility can be related to the Flory-Huggins interaction parameter $(\chi)$ or to the interfacial energy $(\gamma)$ between the components. In $\mathrm{D}_{1}: \mathrm{D}_{2}: \mathrm{A}$ ternary blends in which $\mathrm{D}_{1}$ (PDPP3T) and $\mathrm{D}_{2}$ (FTAZ) were polymers, a large negative (that is, attractive) $\chi$ between $\mathrm{D}_{1}$ and $\mathrm{D}_{2}$ was found to be detrimental to the $\mathrm{PCE}^{114}$. The ternary cell produced a lower photocurrent than stratified binary cells $\left(\mathrm{D}_{1}: \mathrm{A}\right.$ and $\left.\mathrm{D}_{2}: \mathrm{A}\right)$ prepared by a sequential casting technique as a result of poorer hole transport. This effect was attributed to the formation of a homogeneous solid-solution donor phase owing to the mutual affinity of 
the two polymers, which increases the tortuosity of the charge-transport paths and thereby lowers hole mobility. In $\mathrm{D}: \mathrm{A}_{1}: \mathrm{A}_{2}$ cells in which the acceptors were small molecules (non-fullerenes), the role of the interfacial tension between the acceptors and the donor on phase separation and the $V_{\text {oc }}$ has been explored. ${ }^{115}$ In this example, the donor was a polymer that exhibits strong temperature-dependent aggregation behaviour (PTFB-O), and the resultant donor aggregates served as scaffolds around which the acceptors arranged. The large $\gamma$ between the acceptors and the donor ensured strong donor-acceptor phase separation. Conversely, the low $\gamma$ between the acceptors enabled them to form a homogeneous solution phase. Contrary to the $\mathrm{D}_{1}: \mathrm{D}_{2}$ :A case, for the $\mathrm{D}: \mathrm{A}_{1}: \mathrm{A}_{2}$ cells, the presence of the mixed phase increased performance compared with blends in which there was a large $\gamma$ between the acceptors. Structural characterization by X-ray diffraction revealed that the introduction of $\mathrm{A}_{2}$ (IEIC-Th) enhanced the crystallization of $\mathrm{A}_{1}$ (ITIC-Th), which decreased the rate of charge recombination and improved charge transport and collection. These two examples suggest that mixing of two donors or acceptors in a ternary blend is usually detrimental but can occasionally be favourable if these materials exhibit 'physical compatibility', that is, if the structural order is either unperturbed or even improved for enhanced charge collection upon addition of the third component. These examples also demonstrate that many thermodynamic parameters come into play in guiding the formation of the ternary nanomorphology, which is, however, ultimately dictated by the transformation kinetics imposed through processing. These complexities have made it difficult to answer unequivocally the central morphological question: is it preferable for the two donors or acceptors to mix and form a solid solution, or is their phase separation more advantageous in optimizing the PCE?

Currently, there is no consensus regarding which structure ultimately leads to the highest performance as the results remain system-dependent. DR3TBDTT:DR3TBDTT-E:PC $70 B_{1}\left(\mathrm{D}_{2}: \mathrm{D}_{1}: \mathrm{A}\right)$ cells were studied to explore this question ${ }^{88}$. The IP and EA levels of the neat materials resulted in the formation of type II heterojunctions in both DR3TBDTT:DR3TBDTT-E and DR3TBDTT-E:PC 70 BM, which thus creates an energy cascade in the DR3TBDTT:DR3TBDTT-E:PC 70 BM sequence ${ }^{88}$. The ascast ternary cells exhibited a mixed donor phase, but upon a brief period of vapour annealing in chloroform, the donors phase separated to give a $\mathrm{D}_{2}: \mathrm{D}_{1}$ :A microstructure. Solvent annealing was also found to increase the crystallinity and the crystalline coherence length of the donor polymers. The PCE increase upon annealing (from $8.48 \%$ to $10.26 \%$, Table 1) was attributed to reduced bimolecular recombination owing to the cascade morphology and improved transport through the ordered pure DR3TBDTT donor phase. Indeed, the annealed cells exhibited a higher FF and $J_{\text {sc }}$ compared with the 
as-cast counterparts. The $V_{\mathrm{oc}}$ of the ternary cell, however, was lower than that of DR3TBDTT:PC $70 \mathrm{BM}$ because charges were generated at the DR3TBDTT-E:PC ${ }_{70} \mathrm{BM}$ interface, which has a smaller IP - EA gap. In another example, the PTB7:Si-PCPDTBT:PC $70 \mathrm{BM}$ blend with the best performance contained $15 \mathrm{wt} \%$ of Si-PCPDTBT in the donor phase ${ }^{65}$. At this composition, Si-PCPDTBT did not generate a notable photocurrent. Yet, the PCE increased from $7.52 \%$ for the $\mathrm{PTB} 7: \mathrm{PC}_{70} \mathrm{BM}$ blend to $8.6 \%$ in the ternary blend (Table 1). Again, the increase in PCE was due to an increase in both $J_{\mathrm{sc}}$ and FF, the latter reaching a high value of $77 \%$. An increase in charge-carrier lifetime owing to reduced recombination was also observed. Furthermore, the recombination was nearly exclusively bimolecular, suggesting that the introduction of Si-PCPDTBT led to passivation of electronic traps in PTB7 (Fig. 4c). It was therefore postulated that holes are funnelled from PTB7 to Si-PCPDTBT and thus away from the acceptor, thereby reducing recombination. In addition, it was suggested that transport in Si-PCPDTBT is higher as it is more ordered than nearly amorphous PTB7. In the PTB7:Si-PCPDTBT:PC 70 BM-based cells, there is a small $(30 \mathrm{meV})$ decrease in $V_{\text {oc }}$ from $0.73 \mathrm{~V}$ to $0.70 \mathrm{~V}$. This result confirms that the voltage is determined largely at the donor/acceptor interface at which charges are generated (PTB7:PC $70 \mathrm{BM}$ ) and not by the transport material (Si-PCPDTBT). These observations are in line with the known properties of $\left(\mathrm{D}_{1}, \mathrm{D}_{2}\right)$ :A pseudo-binary interfaces, at which $\mathrm{D}_{1}$ and $\mathrm{D}_{2}$ form a mixed solid solution that gives rise to a pseudo-binary cell in which the donor is the $\left(D_{1}, D_{2}\right)$ homogeneous phase; analogous behaviour is observed for $\mathrm{D}:\left(\mathrm{A}_{1}, \mathrm{~A}_{2}\right)$ pseudo-binary interfaces.

A hallmark of the formation of a homogeneous single phase between $D_{1}$ and $D_{2}$ is the fact that in $\left(D_{1}\right.$, $\mathrm{D}_{2}$ ):A ternary cells, the $V_{\text {oc }}$ can be continuously tuned between that of the two binary components. Although widely observed, the origin of this tunability is not fully understood and is probably attributable to a combination of orbital overlap, increased disorder and effective polarizability. Indeed, the $V_{\mathrm{oc}}$ depends on the local energetic landscape at the donor/acceptor interface and the energy of the charge-transfer exciton. At the molecular level, the energetic landscape depends on the quantum mechanical interactions between the components. At the meso-scale level, disorder also affects the energetic landscape ${ }^{116}$. Additionally, owing to its dipole moment, the energy of the charge-transfer exciton depends on the local dielectric constant, which is dictated by the polarizability. Precise $V_{\text {oc }}$ tunability is an advantage of the pseudo-binary microstructure over the $\mathrm{D}_{1}$ : $\mathrm{A}$ and $\mathrm{D}_{2}$ : $\mathrm{A}$ microstructures. Indeed, in both examples reported above, in which the addition of the third component increases the PCE, no additional photocurrent was produced by the third component. When the purpose of the addition of the third component is to make the cell panchromatic (that is, to expand its absorption 
spectrum), the $V_{\mathrm{oc}}$ is predominantly determined by the lowest-energy charge-transfer state present in the active layer, as higher-energy charge-transfer excitons migrate to those sites. As a result, in microstructures with parallel independent binary junctions, the increase in photocurrent comes at the expense of a large penalty in $V_{\mathrm{oc}}$ - a $V_{\mathrm{oc}}$ close to that of the binary cell with the lowest $V_{\mathrm{oc}}$ would be observed. By contrast, in pseudo-binary blends, the full range of intermediate voltages is accessible, with the actual $V_{\text {oc }}$ depending on composition. Thus, in principle, pseudo-binary morphologies can, at least partially, simultaneously take advantage of the added photocurrent generated by the broader absorption and of the voltage of the higher $V_{\mathrm{oc}}$ binary component. This mechanism arises in P3HT:IDTBR:IDFBR ternary cells, for which differential scanning calorimetry measurements showed that IDFBR is miscible in P3HT, whereas the IDTBR phase separates into crystalline domains at higher compositions $^{35}$. The best-performing cell exhibited a higher integrated EQE than the binary cells and a $V_{\mathrm{oc}}$ of $0.82 \mathrm{~V}$, which is $0.1 \mathrm{~V}$ higher than that of the binary component with the lowest $V_{\text {oc. An }}$ additional $V_{\mathrm{oc}}$ boost was given by the increased disorder of P3HT owing to its miscibility with the acceptors. The result was the highest-efficiency P3HT cell reported to date $(\mathrm{PCE}=7.7 \%)$. Similarly, a tunable $V_{\mathrm{oc}}$ has been observed when using miscible small-molecule acceptors ${ }^{115}$. The main drawback of the pseudo-binary morphology is that the mixed phases typically have poorer charge-transport and charge-recombination properties compared with pure phases and therefore exhibit lower FF and/or $J_{\mathrm{sc}}$ values. In one approach, this problem was circumvented by obtaining a nanomorphology in which some pure crystalline IDTBR was present and phase separated from the donor. The larger EA of IDTBR ensured that the main transport path for electrons was through a crystalline phase that was separated from the donor, thus also reducing recombination. By contrast, in the blends with smallmolecule acceptors, the enhanced crystallinity of the acceptor phase upon blending mitigated the expected degradation in charge transport and FF. This issue was solved in a different TSC by blending structurally similar small-molecule acceptors, which led to a FF of $>78 \%$ owing to a dual effect of increased crystallinity and domain size and/or purity ${ }^{117}$. In another example, a combination of a fullerene acceptor and NFA was used. In this blend, the phase separation of the donor and the NFA in the TSC is smaller than that of the donor:NFA binary, and the binary domains fit into the network formed by the fullerene acceptor $\left(\mathrm{PC}_{70} \mathrm{BM}\right)$. This configuration facilitates charge transport through an energy cascade alignment, which enables an active-layer thickness of $>300 \mathrm{~nm}$ along with a PCE of $13.2 \%{ }^{118}$. 
The examples described above provide some insight into how the morphology of ternary cells affects their performance. If the third component does not expand the absorption of the cell, its sole purpose is to help with charge separation through the formation of an energy cascade. In this case, the third component should be located primarily at the photogenerating interface to leave pure phases, which exhibit a higher degree of order for charge transport and ensure effective suppression of recombination. Because disorder at the donor/acceptor interface typically increases the photovoltage, some limited miscibility between the three components at the interface can be beneficial by creating additional disorder. Indeed, an ideal $\mathrm{TSC}^{88}$ is believed to have a phase-separated energy cascade structure and shows a slightly elevated $V_{\mathrm{oc}}$ compared with the binary cell, which is probably due to limited intermixing between the components at the interface. By contrast, if the purpose of the addition of the third component is to make the cell panchromatic, most of the photogenerating interface should be a mixed phase that contains the three components. A mixed phase will ensure a higher voltage owing to the well-known voltage tuning of pseudo-binary blends. Away from the interface, however, phases should be made of the main absorbers, which should be as pure as possible to enable efficient charge transport and reduced recombination.

\section{[H2] Stability in active-layer materials.}

The introduction of NFAs into BHJ devices has required re-evaluation of the possible mechanisms that lead to instability under environmental conditions relevant to operation ${ }^{32}$. The performance of both binary and ternary devices can degrade as a result of exposure to ambient species such as water; irradiation, particularly in the presence of oxygen; and morphological changes as a result of postdeposition molecular diffusion ${ }^{119-122}$. Ingress of ambient water and oxygen into the active layer can be very detrimental to device stability. Thermodynamically favourable redox reactions can occur depending on the NFA energy levels and orbital energy distributions ${ }^{123}$. In addition, the two most common end groups in state-of-the-art NFAs, rhodanine and indanedione derivatives, are coupled to the electron-rich core via Knoevenagel condensation, which affords an exocyclic conjugated double bond that is potentially susceptible to Michael addition from soft nucleophiles. It is therefore necessary to sterically shield this double bond as much as possible in the molecular design. The mechanistic pathways for photo-oxidation involve two main processes. One process involves exothermic energy transfer from the triplet state of either the donor or the acceptor (formed from intersystem crossing of 
photoexcited singlet excitons) to molecular oxygen, which creates a highly reactive singlet oxygen species $^{124}$. Singlet oxygen can then undergo a range of chemical reactions within the active layer, leading to degradation of the conjugated electronic structure of the organic molecules. In particular, concerted Diels-Alder addition to electron-rich diene units present in the aromatic framework of the active chromophore and alpha-hydrogen abstraction reactions on aliphatic side groups, leading to the formation of hydroxy radicals and subsequent ring-opening reactions, are both plausible mechanisms. The likelihood of these reactions occurring is directly related to the choice of active materials; however almost all NFAs have functional groups that may facilitate these reactions. The second, and more prominent, process involves direct electron transfer from the NFA to molecular oxygen, affording highly reactive superoxide radical anion species ${ }^{125}$. This electrochemical reaction is thermodynamically favourable when the EA of the NFA is shallower (versus vacuum) than the EA of molecular oxygen (3.75 eV versus vacuum). This pathway is of course directly relevant to NFA molecular design, for which it is possible to optimize the energy levels to obtain large $V_{\text {oc }}$ values through shallow LUMO energy levels (versus vacuum). In these cases, the voltage can be optimized at the expense of stability unless there is a significant electrochemical overpotential. Superoxide species can undergo a range of fragmentation, addition and oxidative reactions with the common units found in both polymer acceptors and NFAs, such as thienyls. It is, however, possible to cascade high-energy electrons in a ternary system from a shallow LUMO to a deeper-lying LUMO, where electron transfer from the NFA that leads to superoxide formation is thermodynamically unfavourable. In this case, a considerable increase in photo-oxidation stability would be expected in comparison with the binary component with the higher-lying EA.

Morphological instability, including 'burn-in' arises from quenching of the active layer into a non-equilibrium nanostructure during the kinetically driven solution deposition process ${ }^{126}$. The specific optimum blend ratio for a ternary device is typically determined empirically, which involves balancing the competing requirements of charge separation and charge transport. Achieving charge percolation thresholds for both charge carriers on thin-film formation is a minimum requirement of highperformance devices with high current densities. This can be promoted during the phase-segregation processes, which are thermodynamically governed by the binodal composition and/or miscibility gap in an often very complicated phase diagram that can comprise both eutectic and monotectic phase behaviour $^{127}$. In most cases, the quenched nanomorphology (which can be achieved through the introduction of solvent or solid third component additives, as well as thermal annealing) is significantly 
different from the metastable morphology from the phase diagram (owing to the miscibility gap), which results in an inherent morphological instability usually after light and/or oxygen exposure. Ensuring that the mixed domains have a binodal composition enables a metastable state, which leads to improved stability ${ }^{120}$. Ternary blends can exhibit multiple phases depending on the materials used, their thermal behaviour and their specific $\chi$ parameters. These phases can evolve and further segregate after deposition, driven mainly by both high diffusion constants of the small molecules and high $\chi$, that is, thermodynamically favourable demixing. The third component in a ternary system can be exploited to increase thin-film morphological stability through vitrification of the pure, crystalline phases of the other two components, which suppresses ageing-induced crystallization. This effect was observed in a P3HT:IDTBR:IDFBR ternary device, which retained $>80 \%$ of its initial PCE under dark conditions in nitrogen. By contrast, the binary P3HT:IDTBR device retained only $70 \%$ of its initial PCE under the same conditions ${ }^{35}$. The use of a mixture of $\mathrm{A}_{1}: \mathrm{A}_{2}$ acceptors is also a promising approach for achieving high stability under thermal stress. Both fullerene and NFA mixtures have shown high thermal stability compared with the binary cell counterparts ${ }^{128,129}$. The highly stable glassy nanostructure of $\mathrm{D} \mathrm{A}_{1}: \mathrm{A}_{2}$ ternary mixtures increases the configurational entropy upon mixing of the $A_{1}: A_{2}$ components and reduces both the rate of phase separation and crystallization ${ }^{90}$. As a proof of concept, a quaternary BHJ based on $D_{1}: D_{2}: A_{1}: A_{2}$ was designed and found to retain $>72 \%$ of its initial efficiency after 1 year of thermal degradation owing to dual control of phase separation and crystallization ${ }^{87}$. The third component in ternary blends can induce specific molecular interactions in the BHJ, which can suppress molecular diffusion and reduce the $\chi$ values of the corresponding binary systems. In particular, when $\chi$ corresponds to a binodal composition, it allows for efficient charge percolation. Further complications arise when considering the possibility of vertical stratification induced by either surface-directed spinodal decomposition or surface energy differences between the three components. It is likely that in reports of increased stability of ternary blends, when compared to their binary counterparts, it has been possible to exploit compositions close to the thermodynamic equilibrium and minimize excessive postdeposition crystallization and diffusion, while also ensuring that the components that vertically segregate to the interfaces do not block charge extraction. On reviewing the ternary literature, it seems that most stability improvements are due to a more stable morphology rather than increased resistance to photo-oxidation. Although this is an encouraging potential advantage of ternary systems, enhanced stability seems to occur in most cases through trial and error rather than design. This trial-and-error approach is perhaps due to the inherent complications of optimizing a ternary phase diagram to achieve 
metastability on phase separation while also ensuring a charge-percolation threshold that may be either hypereutectic or hypoeutectic.

\section{[H1] Towards scalability}

Although there has been impressive progress in the development of TSCs, transferring small-area hero devices fabricated through a spin-coating method into reliable, large-area devices that can be produced at an industrial scale remains a challenge to be addressed. First, roll-to-roll (R2R) compatible techniques, such as blade, slot-die and wire-bar coating techniques, ideally in air, should be adopted for the fabrication of OSCs with larger areas. Second, the thickness of the active layer in OPV devices is still well below the commonly accepted thickness of 200-300 nm that is required for scaling-up production $^{130,131}$. In one approach to solve this issue, PTB7-Th was introduced as a $\mathrm{D}_{2}$ polymer into a thick $\mathrm{D}_{1}$ :A binary based on PBTZT-stat-BDTT-8:PC ${ }_{70} \mathrm{BM}^{70}$. In addition to the extended absorption window upon addition of PTB7-Th, the ternary blends delivered PCEs of $>11 \%$ (Table 1) together with an active layer thickness of $300 \mathrm{~nm}$. Effective hole transfer from $\mathrm{D}_{2}$ to $\mathrm{D}_{1}$ was observed, which may occur through a dual mechanism of direct excitation of PTB7-Th and energy transfer from PBTZT-statBDTT-8 to PTB7-Th. This robust active layer was also used as a test-bed material for the fabrication of solar modules that achieved a PCE of $>8 \%$ on glass and $7 \%$ on flexible substrates. Similarly, an $\mathrm{A}_{2}$ (ITIC) acceptor was introduced into a thick D: $\mathrm{A}_{1}$ binary based on PDOT:PC ${ }_{70} \mathrm{BM}^{132}$. However, in this case, in addition to the IR harvesting of ITIC (which leads to a higher $J_{\mathrm{sc}}$ ), its lower charge mobility compared with fullerene acceptors could have a detrimental effect on the charge transport. The lowmobility issue was overcome by tuning the $A_{1}: A_{2}$ ratio to reduce fullerene aggregation, which is detrimental for charge transport, leading to a PCE of $>11 \%$ with a thick active layer $(>230 \mathrm{~nm})$. Recently, similar results were obtained by exploring all-polymer TSCs processed in a non-halogenated solvent; these TSCs achieved PCEs of $>8 \%$ and active-layer thicknesses of $>400 \mathrm{~nm}$ (ref. ${ }^{133}$ ). Again, the good miscibility of the $\mathrm{D}_{1}$ and $\mathrm{D}_{2}$ polymers and their high and balanced charge mobilities were the key elements for achieving a high PCE.

\section{Future perspectives}

Future ternary molecular design strategies must address the dual requirements of both efficiency and stability, which will involve optimizing the energy cascade to simultaneously maximize cell voltage 
and ensure broad spectral coverage and charge generation. Moreover, designing universal third components to have intermolecular interactions with the binary components that promote optimal morphologies in the ternary blend could be an approach for optimizing material properties, particularly to reduce post-deposition diffusion and increase stability compared with binary devices. One of the key advantages of TSCs is the improvement in one or more of the photovoltaic parameters of binary devices. Therefore, understanding the limitations of binary devices has crucial implications for the design of ternary blends and devices. Through judicious design of the third component and the resultant improvement in the photovoltaic parameters, it will nearly always be possible to improve upon the performance of a binary cell through the ternary approach.

With the development of NFAs, the OSC field has witnessed an impressive increase in $V_{\text {oc }}$ values, which now exceed $1 \mathrm{~V}$, and the suppression of voltage losses. A decrease in the difference between the radiative $V_{\mathrm{oc}}$ calculated from the Shockley-Queisser limit ( $\left.V_{\mathrm{ocSQ}}\right)$ and the measured $V_{\mathrm{oc}}$ has already been reported using NFAs in binary devices ${ }^{56}$. The $V_{\text {ocSQ }}$ can be estimated from either the absorption edge of the blend by calculating the optical bandgap or from the quantum efficiency spectrum edge, for which the smallest energetic transition is considered. Using a third component that forms an energy cascade alignment with the binary components but has an absorption profile that overlaps with that of the binary components would increase the $V_{\mathrm{oc}}$ without changing the $V_{\mathrm{ocSQ}}$. This strategy would reduce the difference between $V_{\mathrm{ocSQ}}$ and $V_{\mathrm{oc}}$. Furthermore, using a ternary strategy to increase the FF will be crucial in enabling present high-performance materials, which are typically limited to an active layer thickness of $\sim 100 \mathrm{~nm}$, to be processed with thicknesses compatible with high-throughput fabrication methods. Moreover, low-FF binary devices can be re-visited as potential $\mathrm{D}_{1}$ : $\mathrm{A}_{1}$ systems for TSCs, in which the third component would improve the FF by increasing the efficiency of charge generation and extraction through reduced trap-assisted recombination. By combining NFA design with the selection of an appropriate donor to maximize voltage output and the introduction of a second acceptor with favourable charge-transport characteristics to minimize recombination, it is therefore possible to simultaneously optimize all OSC parameters. The nanomorphology of ternary blends is the source of the high performance of TSCs as well as the long-term stability. One of the recent strategies adopted in TSCs to circumvent detrimental morphological issues such as demixing, is the sequential deposition of the binary components and third component to yield a stratified BHJ solar cell ${ }^{114}$. With this method, a pseudo-tandem forms without the need of an intermediate recombination layer, which offers the opportunity to redesign conventional ternary device architectures. 
Impressive progress has been made in TSC device optimization. Considering the individually reported $J_{\mathrm{sc}}\left(>20 \mathrm{~mA} \mathrm{~cm}^{-2}\right), V_{\mathrm{oc}}(>1 \mathrm{~V})$ and FF $(>75 \%)$ values collected in Table 1 for ternary blends, PCEs $>15 \%$ are likely to be achieved in the near future. With stable and large-area solar cells as well as readily scalable processing methods, the implementation of ternary blends into OSCs could serve as a springboard to propel the field of OPVs to commercial realization. However, there remains a need for development of these devices from the laboratory-scale into commercial-scale prototypes. The largearea devices reported to date have low efficiencies, and there are additional challenges, such as the development of proper processing and/or printing techniques and the optimization of printable ink formulations and module design, which make the commercial realization of OPVs an ambitious goal. In order to reduce the time and materials used for the development stage, further progress in the development of facile and non-invasive methods to obtain ternary phase diagrams using spectroscopic tools would be beneficial. Moving towards real-world applications of OPVs, such as indoor applications and transparent solar cells for building-integrated photovoltaics or greenhouses, highthroughput material optimization tools with combinatorial methods are required to reduce the time spent searching for high-performance materials as well as to optimize the resources used. Utilizing machine learning for the design and discovery of the high-performance materials is an emerging approach that can be implemented in the OPV field for the design of optimal third components. Once these issues are addressed, long-term reliability tests of OPV devices and modules with various real-life outdoor conditions need to be explored to move a step closer to commercial OPVs for real-world applications.

\section{Acknowledgements}

The authors acknowledge funding from the King Abdullah University of Science and Technology (KAUST) and thank the UK Engineering and Physical Sciences Research Council (EPSRC) for financial support (Project EP/G037515/1, EP/M005143/1, ECFP7 and Project SC2 (610115)). A.S. acknowledges support from the US National Science Foundation (CBET Award no. 1510481).

\section{Author contributions}

D.B. and N.G researched data for the article. A.S. wrote the morphology-related sections. I.M. wrote the sections on chemical design and stability. N.G. wrote the charge transport and recombination section. D.B. wrote the section on operating modes of TSCs, energetics and the introduction. All authors discussed, edited and reviewed the article prior to submission. 


\section{References}

1. De Gennes, P.-G. Recherches sur les effets de la radiation chimique de la lumiere solaire au moyen des courants electriques. Comptes Rendus L'Academie des Sci. 9, 145-149 (1839).

2. Kepler, R. G. Charge carrier production and mobility in anthracene crystals. Phys. Rev. 119, 1226-1229 (1960).

3. Green, M. A. \& Bremner, S. P. Energy conversion approaches and materials for high-efficiency photovoltaics. Nat. Mater. 16, 23-34 (2016).

4. Brédas, J.-L., Sargent, E. H. \& Scholes, G. D. Photovoltaic concepts inspired by coherence effects in photosynthetic systems. Nat. Mater. 16, 35-44 (2016).

5. McCulloch, I., Salleo, A. \& Chabinyc, M. Avoid the kinks when measuring mobility. Science 352, 1521-1522 (2016).

6. Gómez-Bombarelli, R. et al. Design of efficient molecular organic light-emitting diodes by a high-throughput virtual screening and experimental approach. Nat. Mater. 15, 1120-1127 (2016).

7. Chu, S., Cui, Y. \& Liu, N. The path towards sustainable energy. Nat. Mater. 16, 16-22 (2016).

8. Baeg, K.-J., Binda, M., Natali, D., Caironi, M. \& Noh, Y.-Y. Organic Light Detectors: Photodiodes and Phototransistors. Adv. Mater. 25, 4267-4295 (2013).

9. Ren, X. et al. Organic Field-Effect Transistor for Energy-Related Applications: Low-PowerConsumption Devices, Near-Infrared Phototransistors, and Organic Thermoelectric Devices. Adv. Energy Mater. 8, 1801003 (2018).

10. Leo, K. Organic photovoltaics. Nat. Rev. Mater. 1, 16056 (2016).

11. Brabec, C. J., Sariciftci, N. S. \& Hummelen, J. C. Plastic Solar Cells. Adv. Funct. Mater. 11, 1526 (2001).

12. Günes, S., Neugebauer, H. \& Sariciftci, N. S. Conjugated Polymer-Based Organic Solar Cells. Chem. Rev. 107, 1324-1338 (2007).

13. Deibel, C. \& Dyakonov, V. Polymer-fullerene bulk heterojunction solar cells. Reports Prog. Phys. 73, 096401 (2010).

14. Li, G., Zhu, R. \& Yang, Y. Polymer solar cells. Nat. Photonics 6, 153-161 (2012).

15. Lu, L. et al. Recent Advances in Bulk Heterojunction Polymer Solar Cells. Chem. Rev. 115, 12666-12731 (2015). 
16. Kang, H. et al. Bulk-Heterojunction Organic Solar Cells: Five Core Technologies for Their Commercialization. Adv. Mater. 28, 7821-7861 (2016).

17. Inganäs, O. Organic Photovoltaics over Three Decades. Adv. Mater. 30, 1800388 (2018).

18. Lucera, L. et al. Highly efficient, large area, roll coated flexible and rigid OPV modules with geometric fill factors up to $98.5 \%$ processed with commercially available materials. Energy Environ. Sci. 9, 89-94 (2015).

19. Søndergaard, R. R., Hösel, M. \& Krebs, F. C. Roll-to-Roll fabrication of large area functional organic materials. J. Polym. Sci. Part B Polym. Phys. 51, 16-34 (2013).

20. Po, R. et al. From lab to fab: how must the polymer solar cell materials design change? - an industrial perspective. Energy Environ. Sci. 7, 925 (2014).

21. Tang, C. W. Two-layer organic photovoltaic cell. Appl. Phys. Lett. 48, 183-185 (1986).

22. Yu, G., Gao, J., Hummelen, J. C., Wudl, F. \& Heeger, A. J. Polymer Photovoltaic Cells: Enhanced Efficiencies via a Network of Internal Donor-Acceptor Heterojunctions. Science 270, 1789-1791 (1995).

23. Halls, J. J. M. et al. Efficient photodiodes from interpenetrating polymer networks. Nature 376, 498-500 (1995).

24. Liang, Y. et al. For the bright future-bulk heterojunction polymer solar cells with power conversion efficiency of 7.4\%. Adv. Mater. 22, 135-138 (2010).

25. He, Z. et al. Single-junction polymer solar cells with high efficiency and photovoltage. Nat. Photonics 9, 174-179 (2015).

26. Liu, Y. et al. Aggregation and morphology control enables multiple cases of high-efficiency polymer solar cells. Nat. Commun. 5, 5293 (2014).

27. Liu, J. et al. Fast charge separation in a non-fullerene organic solar cell with a small driving force. Nat. Energy 1, 16089 (2016).

28. Zhao, W. et al. Fullerene-Free Polymer Solar Cells with over $11 \%$ Efficiency and Excellent Thermal Stability. Adv. Mater. 28, 4734-4739 (2016).

29. Sharenko, A. et al. A High-Performing Solution-Processed Small Molecule : Perylene Diimide Bulk Heterojunction Solar Cell. Adv. Mater. 25, 4403-4406 (2013).

30. Kumari, T., Lee, S. M., Kang, S., Chen, S. \& Yang, C. Ternary solar cells with a mixed face-on and edge-on orientation enable an unprecedented efficiency of 12.1\%. Energy Environ. Sci. 10, 258-265 (2017).

31. Thompson, B. C. \& Fréchet, J. M. J. Polymer-Fullerene Composite Solar Cells. Angew. Chemie 
Int. Ed. 47, 58-77 (2008).

32. Gasparini, N. et al. The Physics of Small Molecule Acceptors for Efficient and Stable Bulk Heterojunction Solar Cells. Adv. Energy Mater. 8, 1703298 (2018).

33. Wadsworth, A. et al. Critical review of the molecular design progress in non-fullerene electron acceptors towards commercially viable organic solar cells. Chem. Soc. Rev. (2019). doi:10.1039/C7CS00892A

34. Baran, D. et al. Robust nonfullerene solar cells approaching unity external quantum efficiency enabled by suppression of geminate recombination. Nat. Commun. 9, 2059 (2018).

35. Baran, D. et al. Reducing the efficiency-stability-cost gap of organic photovoltaics with highly efficient and stable small molecule acceptor ternary solar cells. Nat. Mater. 16, 363-369 (2017).

This paper introduces a universal strategy for achieving highly efficient and stable smallmolecule acceptor TSCs.

36. Holliday, S. et al. High-efficiency and air-stable P3HT-based polymer solar cells with a new non-fullerene acceptor. Nat. Commun. 7, 11585 (2016).

37. Hou, J., Inganäs, O., Friend, R. H. \& Gao, F. Organic solar cells based on non-fullerene acceptors. Nat. Mater. 17, 119-128 (2018).

38. Zhang, J., Tan, H. S., Guo, X., Facchetti, A. \& Yan, H. Material insights and challenges for nonfullerene organic solar cells based on small molecular acceptors. Nat. Energy 3, 720-731 (2018).

39. Nielsen, C. B., Holliday, S., Chen, H.-Y., Cryer, S. J. \& McCulloch, I. Non-Fullerene Electron Acceptors for Use in Organic Solar Cells. Acc. Chem. Res. 48, 2803-2812 (2015).

40. Holliday, S., Li, Y. \& Luscombe, C. K. Recent advances in high performance donor-acceptor polymers for organic photovoltaics. Prog. Polym. Sci. 70, 34-51 (2017).

41. Holliday, S. et al. A Rhodanine Flanked Nonfullerene Acceptor for Solution-Processed Organic Photovoltaics. J. Am. Chem. Soc. 137, 898-904 (2015).

42. Yao, H. et al. Design and Synthesis of a Low Bandgap Small Molecule Acceptor for Efficient Polymer Solar Cells. Adv. Mater. 28, 8283-8287 (2016).

43. Hwang, Y.-J., Earmme, T., Courtright, B. a. E., Eberle, F. N. \& Jenekhe, S. a. n-Type Semiconducting Naphthalene Diimide-Perylene Diimide Copolymers: Controlling Crystallinity, Blend Morphology, and Compatibility Toward High-Performance All-Polymer Solar Cells. J. Am. Chem. Soc. 137, 4424-4434 (2015).

44. Mu, C. et al. High-Efficiency All-Polymer Solar Cells Based on a Pair of Crystalline LowBandgap Polymers. Adv. Mater. 26, 7224-7230 (2014). 
45. Zhou, Y. et al. High Performance All-Polymer Solar Cell via Polymer Side-Chain Engineering. Adv. Mater. 26, 3767-3772 (2014).

46. Fan, B. et al. All-Polymer Solar Cells Based on a Conjugated Polymer Containing SiloxaneFunctionalized Side Chains with Efficiency over 10\%. Adv. Mater. 29, 1703906 (2017).

47. Zheng, Z. et al. A Highly Efficient Non-Fullerene Organic Solar Cell with a Fill Factor over 0.80 Enabled by a Fine-Tuned Hole-Transporting Layer. Adv. Mater. 30, 1801801 (2018).

48. Ramirez, I., Causa', M., Zhong, Y., Banerji, N. \& Riede, M. Key Tradeoffs Limiting the Performance of Organic Photovoltaics. Adv. Energy Mater. 8, 1703551 (2018).

49. Wright, M., Lin, R., Tayebjee, M. J. Y. \& Conibeer, G. Effect of Blend Composition on Bulk Heterojunction Organic Solar Cells: A Review. Sol. RRL 1, 1700035 (2017).

50. Peters, C. H. et al. High Efficiency Polymer Solar Cells with Long Operating Lifetimes. Adv. Energy Mater. 1, 491-494 (2011).

51. Ryno, S. M., Ravva, M. K., Chen, X., Li, H. \& Brédas, J.-L. Molecular Understanding of Fullerene - Electron Donor Interactions in Organic Solar Cells. Adv. Energy Mater. 7, 1601370 (2017).

52. Gasparini, N. et al. Burn-in Free Nonfullerene-Based Organic Solar Cells. Adv. Energy Mater. 7, 1700770 (2017).

53. Gasparini, N. et al. Polymer:Nonfullerene Bulk Heterojunction Solar Cells with Exceptionally Low Recombination Rates. Adv. Energy Mater. 7, 1701561 (2017).

54. Liu, Y., Zuo, L., Shi, X., Jen, A. K.-Y. \& Ginger, D. S. Unexpectedly Slow Yet Efficient Picosecond to Nanosecond Photoinduced Hole-Transfer Occurs in a Polymer/Nonfullerene Acceptor Organic Photovoltaic Blend. ACS Energy Lett. 3, 2396-2403 (2018).

55. Qian, D. et al. Design rules for minimizing voltage losses in high-efficiency organic solar cells. Nat. Mater. 17, 703-709 (2018).

56. Baran, D. et al. Reduced voltage losses yield 10\% efficient fullerene free organic solar cells with >1 V open circuit voltages. Energy Environ. Sci. 9, 3783-3793 (2016).

57. Meng, L. et al. Organic and solution-processed tandem solar cells with $17.3 \%$ efficiency. Science 361, 1094-1098 (2018).

58. Zhang, K. et al. 11.2\% All-Polymer Tandem Solar Cells with Simultaneously Improved Efficiency and Stability. Adv. Mater. 30, 1803166 (2018).

59. Yuan, J. et al. Improved Tandem All-Polymer Solar Cells Performance by Using Spectrally Matched Subcells. Adv. Energy Mater. 8, 1703291 (2018). 
60. Qin, Y. et al. Achieving 12.8\% Efficiency by Simultaneously Improving Open-Circuit Voltage and Short-Circuit Current Density in Tandem Organic Solar Cells. Adv. Mater. 29, 1606340 (2017).

61. Li, N. \& Brabec, C. J. Air-processed polymer tandem solar cells with power conversion efficiency exceeding 10\%. Energy Environ. Sci. 8, 2902-2909 (2015).

62. Ameri, T., Li, N. \& Brabec, C. J. Highly efficient organic tandem solar cells: a follow up review. Energy Environ. Sci. 6, 2390-2413 (2013).

63. Guo, F. et al. A generic concept to overcome bandgap limitations for designing highly efficient multi-junction photovoltaic cells. Nat. Commun. 6, 7730 (2015).

64. Spyropoulos, G. D. et al. Flexible organic tandem solar modules with $6 \%$ efficiency: combining roll-to-roll compatible processing with high geometric fill factors. Energy Environ. Sci. 7, 32843290 (2014).

65. Gasparini, N. et al. Designing ternary blend bulk heterojunction solar cells with reduced carrier recombination and a fill factor of 77\%. Nat. Energy 1, 16118 (2016).

This paper demonstrates how trap-assisted recombination in binary blends can be reduced by adding a cystalline third component, leading to high FF values in ternary blends.

66. Huang, W., Cheng, P., Yang, Y. M., Li, G. \& Yang, Y. High-Performance Organic BulkHeterojunction Solar Cells Based on Multiple-Donor or Multiple-Acceptor Components. Adv. Mater. 30, 1705706 (2018).

67. Lu, L., Kelly, M. A., You, W. \& Yu, L. Status and prospects for ternary organic photovoltaics. Nat. Photonics 9, 491-500 (2015).

68. An, Q. et al. Versatile ternary organic solar cells: a critical review. Energy Environ. Sci. 9, 281322 (2016).

69. Ameri, T., Khoram, P., Min, J. \& Brabec, C. J. Organic ternary solar cells: A review. Adv. Mater. 25, 4245-4266 (2013).

70. Gasparini, N. et al. High-performance ternary organic solar cells with thick active layer exceeding 11\% efficiency. Energy Environ. Sci. 10, 885-892 (2017).

71. Ameri, T. et al. Performance Enhancement of the P3HT/PCBM Solar Cells through NIR Sensitization Using a Small-Bandgap Polymer. Adv. Energy Mater. 2, 1198-1202 (2012).

72. Ma, X. et al. Efficient Ternary Polymer Solar Cells with Two Well-Compatible Donors and One Ultranarrow Bandgap Nonfullerene Acceptor. Adv. Energy Mater. 8, 1702854 (2018).

73. Cheng, P., Li, Y. \& Zhan, X. Efficient ternary blend polymer solar cells with indene-C60 
bisadduct as an electron-cascade acceptor. Energy Environ. Sci. 7, 2005-2011 (2014).

74. Street, R. a., Davies, D., Khlyabich, P. P., Burkhart, B. \& Thompson, B. C. Origin of the tunable open-circuit voltage in ternary blend bulk heterojunction organic solar cells. J. Am. Chem. Soc.

135, 986-989 (2013).

\section{This paper introduces the alloy model in TSCs.}

75. Mollinger, S. A., Vandewal, K. \& Salleo, A. Microstructural and Electronic Origins of OpenCircuit Voltage Tuning in Organic Solar Cells Based on Ternary Blends. Adv. Energy Mater. 5, 1501335 (2015).

76. Liu, S. et al. Enhanced efficiency of polymer solar cells by adding a high-mobility conjugated polymer. Energy Environ. Sci. 8, 1463-1470 (2015).

77. Zhang, M. et al. Efficient ternary non-fullerene polymer solar cells with PCE of $11.92 \%$ and FF of 76.5\%. Energy Environ. Sci. 11, 841-849 (2018).

78. Yang, Y. (Michael) et al. High-performance multiple-donor bulk heterojunction solar cells. Nat. Photonics 9, 190-198 (2015).

\section{This paper provides design rules for fabricating high-performance multiple-donor BHJ solar cells.}

79. Yu, R., Yao, H. \& Hou, J. Recent Progress in Ternary Organic Solar Cells Based on Nonfullerene Acceptors. Adv. Energy Mater. 8, 1702814 (2018).

80. Li, H., Lu, K. \& Wei, Z. Polymer/Small Molecule/Fullerene Based Ternary Solar Cells. Adv. Energy Mater. 1602540, 1602540 (2017).

81. Zhao, W., Li, S., Zhang, S., Liu, X. \& Hou, J. Ternary Polymer Solar Cells based on Two Acceptors and One Donor for Achieving 12.2\% Efficiency. Adv. Mater. 29, 1604059 (2017).

82. Xu, X. et al. Highly Efficient Ternary-Blend Polymer Solar Cells Enabled by a Nonfullerene Acceptor and Two Polymer Donors with a Broad Composition Tolerance. Adv. Mater. 29, 1704271 (2017).

83. Lu, H. et al. Ternary-Blend Polymer Solar Cells Combining Fullerene and Nonfullerene Acceptors to Synergistically Boost the Photovoltaic Performance. Adv. Mater. 28, 9559-9566 (2016).

84. Gupta, V., Bharti, V., Kumar, M., Chand, S. \& Heeger, A. J. Polymer-Polymer Förster Resonance Energy Transfer Significantly Boosts the Power Conversion Efficiency of BulkHeterojunction Solar Cells. Adv. Mater. 27, 4398-4404 (2015).

85. Lu, L., Xu, T., Chen, W., Landry, E. S. \& Yu, L. Ternary blend polymer solar cells with 
enhanced power conversion efficiency. Nat. Photonics 8, 716-722 (2014).

86. Lu, L., Chen, W., Xu, T. \& Yu, L. High-performance ternary blend polymer solar cells involving both energy transfer and hole relay processes. Nat. Commun. 6, 7327 (2015).

This paper reports highly efficient ternary blends that exhibit both charge and energy transfer mechanisms

87. Nam, M. et al. Long-term efficient organic photovoltaics based on quaternary bulk heterojunctions. Nat. Commun. 8, 14068 (2017).

88. Wang, Z. et al. From Alloy-Like to Cascade Blended Structure: Designing High-Performance All-Small-Molecule Ternary Solar Cells. J. Am. Chem. Soc. 140, 1549-1556 (2018).

89. Zhang, J. et al. Conjugated polymer-small molecule alloy leads to high efficient ternary organic solar cells. J. Am. Chem. Soc. 137, 8176-8183 (2015).

90. de Zerio, A. D. \& Müller, C. Glass Forming Acceptor Alloys for Highly Efficient and Thermally Stable Ternary Organic Solar Cells. Adv. Energy Mater. 8, 1702741 (2018).

91. Naveed, H. B. \& Ma, W. Miscibility-Driven Optimization of Nanostructures in Ternary Organic Solar Cells Using Non-fullerene Acceptors. Joule 2, 621-641 (2018).

92. Ameri, T. et al. Morphology analysis of near IR sensitized polymer/fullerene organic solar cells by implementing low bandgap heteroanalogue C-/Si-PCPDTBT. J. Mater. Chem. A 2, 1946119472 (2014).

93. Yu, R. et al. Two Well-Miscible Acceptors Work as One for Efficient Fullerene-Free Organic Solar Cells. Adv. Mater. 29, 1700437 (2017).

94. Lee, J. et al. Overcoming Fill Factor Reduction in Ternary Polymer Solar Cells by Matching the Highest Occupied Molecular Orbital Energy Levels of Donor Polymers. Adv. Energy Mater. 8, 1702251 (2018).

95. Xu, W.-L. et al. Förster Resonance Energy Transfer and Energy Cascade in Broadband Photodetectors with Ternary Polymer Bulk Heterojunction. J. Phys. Chem. C 119, 21913-21920 (2015).

96. Koppe, M. et al. Charge carrier dynamics in a ternary bulk heterojunction system consisting of P3HT, fullerene, and a low bandgap polymer. Adv. Energy Mater. 3, 949-958 (2013).

97. Yang, L., Zhou, H., Price, S. C. \& You, W. Parallel-like Bulk Heterojunction Polymer Solar Cells. J. Am. Chem. Soc. 134, 5432-5435 (2011).

\section{This paper introduces the parallel-like model in TSCs.}

98. Khlyabich, P. P., Burkhart, B. \& Thompson, B. C. Efficient ternary blend bulk heterojunction 
solar cells with tunable open-circuit voltage. J. Am. Chem. Soc. 133, 14534-14537 (2011).

99. Cheng, P. et al. Alloy Acceptor: Superior Alternative to PCBM toward Efficient and Stable Organic Solar Cells. Adv. Mater. 28, 8021-8028 (2016).

100. Ke, L. et al. A Series of Pyrene-Substituted Silicon Phthalocyanines as Near-IR Sensitizers in Organic Ternary Solar Cells. Adv. Energy Mater. 6, 1502355 (2016).

101. Xiao, Z., Jia, X. \& Ding, L. Ternary organic solar cells offer $14 \%$ power conversion efficiency. Sci. Bull. 62, 1562-1564 (2017).

102. Zhang, S., Qin, Y., Zhu, J. \& Hou, J. Over 14\% Efficiency in Polymer Solar Cells Enabled by a Chlorinated Polymer Donor. Adv. Mater. 30, 1800868 (2018).

103. Koppe, M. et al. Near IR Sensitization of Organic Bulk Heterojunction Solar Cells: Towards Optimization of the Spectral Response of Organic Solar Cells. Adv. Funct. Mater. 20, 338-346 (2010).

\section{This paper provides the first example of TSCs with enhanced absorption in the NIR region of the solar spectrum.}

104. Liu, T. et al. Ternary Organic Solar Cells Based on Two Highly Efficient Polymer Donors with Enhanced Power Conversion Efficiency. Adv. Energy Mater. 6, 1502109 (2016).

105. Zhao, F. et al. Combining Energy Transfer and Optimized Morphology for Highly Efficient Ternary Polymer Solar Cells. Adv. Energy Mater. 7, 1602552 (2017).

106. Li, W. et al. Contrasting Effects of Energy Transfer in Determining Efficiency Improvements in Ternary Polymer Solar Cells. Adv. Funct. Mater. 28, 1704212 (2018).

107. Zhou, H. et al. High-efficiency polymer solar cells enhanced by solvent treatment. Adv. Mater. 25, 1646-1652 (2013).

108. Song, X. et al. Controlling Blend Morphology for Ultrahigh Current Density in Nonfullerene Acceptor-Based Organic Solar Cells. ACS Energy Lett. 3, 669-676 (2018).

109. He, Y., Chen, H. Y., Hou, J. \& Li, Y. Indene - C60 bisadduct: A new acceptor for highperformance polymer solar cells. J. Am. Chem. Soc. 132, 1377-1382 (2010).

110. Su, W. et al. Efficient ternary blend all-polymer solar cells with a polythiophene derivative as a hole-cascade material. J. Mater. Chem. A 4, 14752-14760 (2016).

111. Sweetnam, S. et al. Characterization of the Polymer Energy Landscape in Polymer:Fullerene Bulk Heterojunctions with Pure and Mixed Phases. J. Am. Chem. Soc. 136, 14078-14088 (2014).

112. Bartelt, J. A. et al. The importance of fullerene percolation in the mixed regions of polymer- 
fullerene bulk heterojunction solar cells. Adv. Energy Mater. 3, 364-374 (2013).

113. Shang, Z. et al. Trade-Off between Trap Filling, Trap Creation, and Charge Recombination Results in Performance Increase at Ultralow Doping Levels in Bulk Heterojunction Solar Cells. Adv. Energy Mater. 6, 1601149 (2016).

114. Ghasemi, M. et al. Panchromatic Sequentially Cast Ternary Polymer Solar Cells. Adv. Mater. 29, 1604603 (2017).

115. Jiang, K. et al. Multiple Cases of Efficient Nonfullerene Ternary Organic Solar Cells Enabled by an Effective Morphology Control Method. Adv. Energy Mater. 8, 1701370 (2018).

116. Poelking, C. et al. Impact of mesoscale order on open-circuit voltage in organic solar cells. Nat. Mater. 14, 434-439 (2015).

117. Liu, T. et al. Use of two structurally similar small molecular acceptors enabling ternary organic solar cells with high efficiencies and fill factors. Energy Environ. Sci. 11, 3275-3282 (2018).

118. Zhou, Z. et al. High-efficiency small-molecule ternary solar cells with a hierarchical morphology enabled by synergizing fullerene and non-fullerene acceptors. Nat. Energy 3, 952959 (2018).

This paper and ref.70 show how the ternary strategy enables the fabrication of thick active layers for high-efficiency and scalable ternary blends.

119. Jørgensen, M. et al. Stability of Polymer Solar Cells. Adv. Mater. 24, 580-612 (2012).

120. Li, N. et al. Abnormal strong burn-in degradation of highly efficient polymer solar cells caused by spinodal donor-acceptor demixing. Nat. Commun. 8, 14541 (2017).

121. Heumueller, T. et al. Morphological and electrical control of fullerene dimerization determines organic photovoltaic stability. Energy Environ. Sci. 9, 247-256 (2015).

122. Hintz, H. et al. Photodegradation of P3HT-A Systematic Study of Environmental Factors. Chem. Mater. 23, 145-154 (2011).

123. de Leeuw, D. M., Simenon, M. M. J., Brown, A. R. \& Einerhand, R. E. F. Stability of n-type doped conducting polymers and consequences for polymeric microelectronic devices. Synth. Met. 87, 53-59 (1997).

124. Salvador, M. et al. Suppressing photooxidation of conjugated polymers and their blends with fullerenes through nickel chelates. Energy Environ. Sci. 10, 2005-2016 (2017).

125. Seemann, A. et al. Reversible and irreversible degradation of organic solar cell performance by oxygen. Sol. Energy 85, 1238-1249 (2011).

126. Peters, C. H. et al. The mechanism of burn-in loss in a high efficiency polymer solar cell. $A d v$. 
Mater. 24, 663-668 (2012).

127. Ye, L. et al. Quantitative relations between interaction parameter, miscibility and function in organic solar cells. Nat. Mater. 17, 253-260 (2018).

128. Diaz de Zerio Mendaza, A. et al. A fullerene alloy based photovoltaic blend with a glass transition temperature above $200{ }^{\circ} \mathrm{C}$. J. Mater. Chem. A 5, 4156-4162 (2017).

129. Su, W. et al. Two compatible nonfullerene acceptors with similar structures as alloy for efficient ternary polymer solar cells. Nano Energy 38, 510-517 (2017).

130. Berny, S. et al. Solar Trees: First Large-Scale Demonstration of Fully Solution Coated, Semitransparent, Flexible Organic Photovoltaic Modules. Adv. Sci. 3, 1500342 (2016).

131. Strohm, S. et al. P3HT: non-fullerene acceptor based large area, semi-transparent PV modules with power conversion efficiencies of $5 \%$, processed by industrially scalable methods. Energy Environ. Sci. 11, 2225-2234 (2018).

132. Zhang, T., Zhao, X., Yang, D., Tian, Y.\& Yang, X. Ternary Organic Solar Cells with \&gt; $\geq 11 \%$ Efficiency Incorporating Thick Photoactive Layer and Nonfullerene Small Molecule Acceptor. Adv. Energy Mater. 8, 1701691 (2018).

133. Fan, B. et al. High-Performance Thick-Film All-Polymer Solar Cells Created Via Ternary Blending of a Novel Wide-Bandgap Electron-Donating Copolymer. Adv. Energy Mater. 8, 1703085 (2018).

134. Hwang, Y. J., Li, H., Courtright, B. A. E., Subramaniyan, S. \& Jenekhe, S. A. Nonfullerene Polymer Solar Cells with 8.5\% Efficiency Enabled by a New Highly Twisted Electron Acceptor Dimer. Adv. Mater. 28, 124-131 (2016).

135. Zhong, L. et al. High Efficiency Ternary Nonfullerene Polymer Solar Cells with Two Polymer Donors and an Organic Semiconductor Acceptor. Adv. Energy Mater. 7, 1602215 (2017).

136. $\mathrm{Su}, \mathrm{W}$. et al. Efficient ternary blend all-polymer solar cells with a polythiophene derivative as a hole-cascade material. J. Mater. Chem. A 4, 14752-14760 (2016).

137. Li, Z. et al. 9.0\% Power Conversion Efficiency From Ternary All-Polymer Solar Cells. Energy Environ. Sci. 10, 2212-2221 (2017).

138. Nian, L. et al. Ternary non-fullerene polymer solar cells with $13.51 \%$ efficiency and a recordhigh fill factor of 78.13\%. Energy Environ. Sci. 11, 3392-3399 (2018).

139. Chen, Y. et al. From Binary to Ternary: Improving the External Quantum Efficiency of SmallMolecule Acceptor-Based Polymer Solar Cells with a Minute Amount of Fullerene Sensitization. Adv. Energy Mater. 7, 1700328 (2017). 
140. Chen, Y. et al. Achieving High-Performance Ternary Organic Solar Cells through Tuning Acceptor Alloy. Adv. Mater. 29, 1603154 (2017).

141. Zhang, H. et al. Improved Domain Size and Purity Enables Efficient All-Small-Molecule Ternary Solar Cells. Adv. Mater. 29, 1703777 (2017).

142. Cheng, P. et al. Realizing Small Energy Loss of $0.55 \mathrm{eV}$, High Open-Circuit Voltage $\geq \$ 1 \mathrm{~V}$ and High Efficiency $\geq \& g t ; 10 \%$ in Fullerene-Free Polymer Solar Cells via Energy Driver. Adv. Mater. 29, 1605216 (2017).

143. Liu, T. et al. Ternary Organic Solar Cells Based on Two Compatible Nonfullerene Acceptors with Power Conversion Efficiency >10\%. Adv. Mater. 28, 10008-10015 (2016).

144. Jiang, W. et al. Ternary Nonfullerene Polymer Solar Cells with $12.16 \%$ Efficiency by Introducing One Acceptor with Cascading Energy Level and Complementary Absorption. Adv. Mater. 30, 1703005 (2018).

Fig. 1 | Chemical structures of widely used donor materials for ternary solar cells. Examples of common donor materials include polymers and small molecules. The representative building blocks for donor materials include thiophene (T) derivatives, benzodithiophene (BDT), benzothiadiazole (BT), thieno[3,4-b]thiophene (TT), benzodithiophene-benzodithiophenedione (BDD) and thieno[3,4c]pyrrole-4,6-dione (TPD).

Fig. 2 Chemical structures of widely used acceptor materials for ternary solar cells. Examples of common used acceptor materials include polymers and small molecules. The representative building blocks for acceptor materials include, [6,6]-phenyl $\mathrm{C}_{61}$-butyric acid methyl ester $\left(\mathrm{PC}_{60} \mathrm{BM}\right),[6,6]-$ phenyl $\mathrm{C}_{71}$-butyric acid methyl ester $\left(\mathrm{PC}_{70} \mathrm{BM}\right)$, indandione and rhodanine derivatives, as well as perylene diimide (PDI), indacenodithiophene (IDT) and indacenodithienothiophene (IDTT) groups.

Fig. 3 | Organic solar cell device architecture and nanomorphology models for BHJ layers. a | Device stack for an organic solar cell comprising charge-collecting electrodes, transport layers and a bulk-heterojunction (BHJ) active layer consisting of a donor and an acceptor. The inset shows the morphology of a general bulk active layer and highlights that there are domains in which no distinct phases (donor or acceptor) exist but rather a mixed morphology is observed. b-f $\mid$ Illustrations showing the possible locations of the third component in a ternary blend. The third component can embed in the donor moiety (panel $\mathbf{b}$ ), embed in the acceptor moiety (panel $\mathbf{c}$ ), reside between the donor and acceptor phases (panel d), co-crystallize with the donor (panel e) or co-crystallize with the acceptor (panel f). 
The regions between the donor and acceptor are shown in white, to make the position of the third component at the interface clearer. ITO, indium tin oxide. Panel $\mathbf{a}$ is adapted with permission from the King Abdullah University of Science and Technology (KAUST) and Ivan Gromicho, Scientific Illustrator at KAUST.

Fig. 4 | The role of the third component in improving the performance of ternary organic solar cells. a | Illustration showing the electronic energy levels of the third component (T) and the binary components (donor $\mathrm{D}_{1}$ and acceptor $\mathrm{A}_{1}$ ) forming an energy cascade within a ternary blend. The formation of an energy cascade alignment contributes to an increase in the open-circuit voltage. b | Absorption profiles of different third components in the UV-vis to near-infrared region. The absorption of the third component can complement that of the binary blend, resulting in an increase in the shortcircuit current. c | Representative illustration showing an ordered third component added to an

amorphous binary donor:acceptor blend. d,e | The introduction of a third component can improve charge transport by enabling trap-free charge transfer in ternary devices (shown for electron transfer in panel d) compared with trap-limited charge transfer in binary devices (shown for electron transfer in panel e).

Fig. $5 \mid J-V$ curves showing the possible improvement in photovoltaic parameters in ternary blends. Schematic representation of the current $(J)$-voltage $(V)$ characteristics of binary and ternary blends. The addition of the third component can increase the open-circuit voltage $\left(V_{\mathrm{oc}}\right)$, short-circuit current $\left(J_{\mathrm{sc}}\right)$ or all the photovoltaic parameters $\left(V_{\mathrm{oc}}, J_{\mathrm{sc}}\right.$ and the fill factor $\left.(\mathrm{FF})\right)$ simultaneously. 
Table 1 | Selected photovoltaic parameters of highly efficient ternary organic solar cells.

\begin{tabular}{|c|c|c|c|c|c|c|c|}
\hline Ternary blend & Type & $\begin{array}{l}V_{\text {oc }} \\
(\mathrm{V})\end{array}$ & $\begin{array}{l}J_{\mathrm{sc}} \\
\left(\mathbf{m A ~ c m} \mathbf{c m}^{-2}\right)\end{array}$ & FF & $\begin{array}{l}\text { PCE } \\
(\%)\end{array}$ & $\begin{array}{l}\text { Relative } \\
\text { increase in } \\
\text { PCE }(\%)^{\mathrm{a}}\end{array}$ & ref. \\
\hline PSEHTT:PTB7-Th:DBFI-EDOT & $\mathrm{D}_{1}: \mathrm{D}_{2}: \mathrm{A}$ & 0.91 & 15.7 & 0.60 & 8.5 & 5 & 134 \\
\hline J51:PTB7-Th:ITIC & $\mathrm{D}_{1}: \mathrm{D}_{2}: \mathrm{A}$ & 0.81 & 17.8 & 0.68 & 9.7 & 9 & 135 \\
\hline PTB7-Th:PBDD-ff4T:PNDI(2OD)-2T & $\mathrm{D}_{1}: \mathrm{D}_{2}: \mathrm{A}$ & 0.82 & 15.7 & 0.56 & 7.2 & 22 & 136 \\
\hline PTB7-Th:PBDTTS-FTAZ:PNDI-T10 & $\mathrm{D}_{1}: \mathrm{D}_{2}: \mathrm{A}$ & 0.84 & 14.4 & 0.74 & 9.0 & 25 & 137 \\
\hline PBDB-T:PTB7-Th:SFBRCN & $\mathrm{D}_{1}: \mathrm{D}_{2}: \mathrm{A}$ & 0.93 & 17.9 & 0.74 & 12.3 & 21 & 82 \\
\hline PTB7-Th:PBDB-T:IEICO-4F & $\mathrm{D}_{1}: \mathrm{D}_{2}: \mathrm{A}$ & 0.74 & 24.1 & 0.65 & 11.6 & 13 & 72 \\
\hline PBDB-T:PDTfBO-TT:ITIC & $\mathrm{D}_{1}: \mathrm{D}_{2}: \mathrm{A}$ & 0.90 & 16.6 & 0.77 & 12.1 & 12 & 138 \\
\hline PBDB-T:PDTfBO-TT:IT-M & $\mathrm{D}_{1}: \mathrm{D}_{2}: \mathrm{A}$ & 0.94 & 18.5 & 0.78 & 13.5 & 15 & \begin{tabular}{ll|}
138 \\
\end{tabular} \\
\hline PBDB-T:ITCPTC:IDT6CN-M & $\mathrm{D}_{1}: \mathrm{D}_{2}: \mathrm{A}$ & 0.88 & 17.4 & 0.77 & 11.9 & 8 & 77 \\
\hline P3HT:PCPDTBT:РC 60 BM & $\mathrm{D}_{1}: \mathrm{D}_{2}: \mathrm{A}$ & 0.57 & 8.1 & 0.63 & 2.8 & 12 & 103 \\
\hline P3HT:Si-PCPDTBT:PC 60 BM & $\mathrm{D}_{1}: \mathrm{D}_{2}: \mathrm{A}$ & 0.59 & 11.0 & 0.62 & 4.0 & 29 & 71 \\
\hline PTB7:Si-PCPDTBT:PC $70 \mathrm{BM}$ & $\mathrm{D}_{1}: \mathrm{D}_{2}: \mathrm{A}$ & 0.70 & 15.9 & 0.77 & 8.6 & 15 & 65 \\
\hline PTB7-Th:Si-PCPDTBT:PC 70 BM & $\mathrm{D}_{1}: \mathrm{D}_{2}: \mathrm{A}$ & 0.76 & 16.8 & 0.71 & 9.1 & 9 & 65 \\
\hline PTB7-Th:p-DTS(FBTTh2) 2: $_{2}$ PC $_{70}$ BM & $\mathrm{D}_{1}: \mathrm{D}_{2}: \mathrm{A}$ & 0.76 & 18.4 & 0.75 & 10.5 & 14 & 89 \\
\hline PBTZT-stat-BDTT-8:PTB7-Th: PC $_{70}$ BM & $\mathrm{D}_{1}: \mathrm{D}_{2}: \mathrm{A}$ & 0.77 & 19.3 & 0.74 & 11.0 & 28 & 70 \\
\hline PTB7:PID2:PC 70 BM & $\mathrm{D}_{1}: \mathrm{D}_{2}: \mathrm{A}$ & 0.72 & 16.8 & 0.68 & 8.2 & 13 & 85 \\
\hline PTB7-Th:PID2: PC70BM & $\mathrm{D}_{1}: \mathrm{D}_{2}: \mathrm{A}$ & 0.78 & 16.7 & 0.71 & 9.2 & 17 & 86 \\
\hline PTB7:PBDTT-SeDPP: PC70BM & $\mathrm{D}_{1}: \mathrm{D}_{2}: \mathrm{A}$ & 0.69 & 18.7 & 0.67 & 8.7 & 36 & 78 \\
\hline PTB7-Th:PDBT-T1: PC $_{70} \mathrm{BM}$ & $\mathrm{D}_{1}: \mathrm{D}_{2}: \mathrm{A}$ & 0.81 & 17.8 & 0.70 & 10.2 & 11 & 104 \\
\hline PTB7-Th:PffBT4T-2OD: PC70BM & $\mathrm{D}_{1}: \mathrm{D}_{2}: \mathrm{A}$ & 0.78 & 19.2 & 0.73 & 10.8 & 15 & 105 \\
\hline DR3TBDTT:DR3TBDTT-E:PC $70 \mathrm{BM}$ & $\mathrm{D}_{1}: \mathrm{D}_{2}: \mathrm{A}$ & 0.90 & 14.97 & 0.77 & 10.3 & 15 & 88 \\
\hline PBDTTT-E-T:IEICO:bis-PC ${ }_{70} \mathrm{BM}$ & $\mathrm{D}: \mathrm{A}_{1}: \mathrm{A}_{2}$ & 0.83 & 18.9 & 0.65 & 10.2 & 23 & 139 \\
\hline PBDTTT-E-T:IEICO: PC $_{70} \mathrm{BM}$ & $\mathrm{D}: \mathrm{A}_{1}: \mathrm{A}_{2}$ & 0.80 & 16.7 & 0.64 & 8.6 & 3 & 139 \\
\hline PBDTTT-E-T:IEICO:IC ${ }_{71} \mathrm{BA}$ & $\mathrm{D}: \mathrm{A}_{1}: \mathrm{A}_{2}$ & 0.83 & 18.9 & 0.65 & 10.2 & 22 & 139 \\
\hline PPBDTBT:ITIC: PC $70 \mathrm{BM}$ & $\mathrm{D}: \mathrm{A}_{1}: \mathrm{A}_{2}$ & 0.89 & 16.7 & 0.68 & 10.4 & 35 & 83 \\
\hline PTB7-Th:TPCE-4PDI:PC $70 \mathrm{BM}$ & $\mathrm{D}: \mathrm{A}_{1}: \mathrm{A}_{2}$ & 0.78 & 17.4 & 0.74 & 10.4 & 9 & 140 \\
\hline PBDB-T:IT-M:bis- $\mathrm{PC}_{70} \mathrm{BM}$ & $\mathrm{D}: \mathrm{A}_{1}: \mathrm{A}_{2}$ & 0.95 & 17.4 & 0.74 & 12.2 & 13 & 81 \\
\hline PDOT:ITIC: PC $_{70} \mathrm{BM}$ & $\mathrm{D}: \mathrm{A}_{1}: \mathrm{A}_{2}$ & 0.96 & 17.5 & 0.67 & 11.2 & 15 & 132 \\
\hline DRTB-T:IDIC: PC $_{70} \mathrm{BM}$ & $\mathrm{D}: \mathrm{A}_{1}: \mathrm{A}_{2}$ & 0.99 & 15.5 & 0.68 & 10.5 & 18 & 141 \\
\hline PTB7-Th:COi8DFIC: PC 70 BM & $\mathrm{D}: \mathrm{A}_{1}: \mathrm{A}_{2}$ & 0.70 & 28.2 & 0.71 & 14.1 & 16 & 101 \\
\hline P3HT:O-IDTBR:IDFBR & $\mathrm{D}: \mathrm{A}_{1}: \mathrm{A}_{2}$ & 0.82 & 14.4 & 0.64 & 7.7 & 22 & 35 \\
\hline J52:IT-M:IEICO & $\mathrm{D}: \mathrm{A}_{1}: \mathrm{A}_{2}$ & 0.85 & 19.7 & 0.67 & 11.1 & 18 & 93 \\
\hline PTB7-Th:IDT-2BR:PDI-2DTT & $\mathrm{D}: \mathrm{A}_{1}: \mathrm{A}_{2}$ & 1.03 & 14.5 & 0.65 & 10.1 & 19 & 142 \\
\hline PDBT-T1:ITIC-Th:SdiPBI-Se & $\mathrm{D}: \mathrm{A}_{1}: \mathrm{A}_{2}$ & 0.93 & 15.4 & 0.70 & 10.3 & 26 & 143 \\
\hline PBDB-T:ITCN:IT-M & $\mathrm{D}: \mathrm{A}_{1}: \mathrm{A}_{2}$ & 0.96 & 17.9 & 0.72 & 12.2 & 12 & 144 \\
\hline PTFB-O:ITIC-Th:IEIC-Th & $\mathrm{D}: \mathrm{A}_{1}: \mathrm{A}_{2}$ & 0.95 & 16.4 & 0.72 & 11.2 & 10 & 115 \\
\hline PTFB-O:ITIC-Th:SF-PDI ${ }_{2}$ & $\mathrm{D}: \mathrm{A}_{1}: \mathrm{A}_{2}$ & 0.95 & 15.1 & 0.73 & 10.5 & 3 & 115 \\
\hline PTB7:IC 60 BA:PC $70 \mathrm{BM}$ & $\mathrm{D}: \mathrm{A}_{1}: \mathrm{A}_{2}$ & 0.72 & 16.3 & 0.69 & 8.2 & 12 & 73 \\
\hline P3HT:SiPc-Py:PC 60 BM & $\mathrm{D}: \mathrm{A}_{1}: \mathrm{A}_{2}$ & 0.65 & 10.0 & 0.63 & 4.1 & 52 & 100 \\
\hline
\end{tabular}


Selected chemical structures of the donors and acceptors are shown in Fig. 1 and Fig. 2, respectively; the remaining structures are shown in the Supplementary Information. $A$ (or $A_{1}$ ) and $D\left(\operatorname{or} D_{1}\right.$ ), primary acceptor or donor, respectively; $A_{2}$ and $D_{2}$, secondary acceptor or donor, respectively; $F F$, fill factor; $J_{s c}$, short-circuit

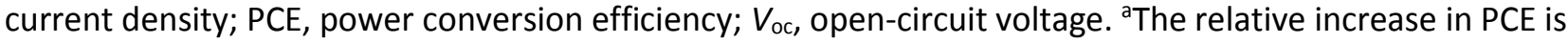
calculated with respect to the binary device $\left(D_{1}: A\right.$ or $\left.D: A_{1}\right)$ with the highest PCE. 